\title{
ON INTERSECTIONS OF RANGES OF PROJECTIONS OF NORM ONE IN BANACH SPACES
}

\author{
T. S. S. R. K. RAO
}

(Communicated by Thomas Schlumprecht)

\begin{abstract}
In this short note we are interested in studying Banach spaces in which the range of a projection of norm one whose kernel is of finite dimension is the intersection of ranges of finitely many projections of norm one whose kernels are of dimension one. We show that for a certain class of Banach spaces $X$, the natural duality between $X$ and $X^{* *}$ can be exploited when the range of the projection is of finite codimension. We show that if $X^{*}$ is isometric to $L^{1}(\mu)$, then any central subspace of finite codimension is an intersection of central subspaces of codimension one. These results extend a recent result of Bandyopadhyay and Dutta which was proved for ranges of projections of norm one with finite dimensional kernel in continuous function spaces and unifies some earlier work of Baronti and Papini.
\end{abstract}

\section{INTRODUCTION}

Let $X$ be a real Banach space. The study of properties of ranges of projections of norm one has always been a central theme in the theory of geometry of Banach spaces. The current study is motivated by some results of Blatter and Cheney [5], Baronti and Papini [4], 3], and the more recent work of Bandyopadhyay and Dutta 2. In these papers for classical Banach spaces such as the space $C(K)$ of continuous functions and the space of integrable functions $L^{1}(\mu)$, the authors give an explicit description of projections of norm one whose kernel is of dimension one and show that any projection of norm one whose kernel is of finite dimension is the intersection of ranges of finitely many projections of norm one whose kernels are of dimension one. Even though proofs of some of these results use specific properties of sequence spaces $c_{0}, \ell^{1}$ and $\ell^{\infty}$, it turns out that the basic duality that exists between abstract $L$-spaces and $M$-spaces and certain types of projections in them can be exploited to obtain similar conclusions. We refer to the monograph by Lacey [11 for basic material on abstract $L$ - and $M$-spaces and Banach spaces $X$ whose dual is isometric to $L^{1}(\mu)$ for some positive measure $\mu$ (the so-called $L^{1}$ predual spaces). Lacey's book also has a detailed study of ranges of projections of norm one on these spaces. In particular we note that the range of a projection of norm one in any of these classes is again a space in the same class. See also the work of Kamińska et al. [10] for the study of ranges of norm one projections in Lorentz sequence space $d(\omega, 1)$ and some subspaces of Musielak-Orlicz sequence spaces equipped with the Luxembourg norm. It should be noted that there are

Received by the editors July 11, 2011 and, in revised form, January 5, 2012.

2010 Mathematics Subject Classification. Primary 47L05; Secondary 46B20, 46 E15.

Key words and phrases. Projections of norm one, finite codimensional subspaces, spaces of continuous functions. 
examples of finite dimensional spaces in which the only projections of norm one are the ones with one dimensional range; see [6]. Examples from [4] show that the intersection of ranges of two projections of norm one, with kernels of dimension one, need not be the range of a projection of norm one.

We next state the three theorems from the work of [4] (Theorem 6.3), 3] and a relevant portion of Theorem 1.1 in 2 that has motivated this work. We recall that $\ell^{\infty^{*}}=\ell^{1} \bigoplus_{1} c_{0}^{\perp}$ so that any $f \in \ell^{\infty^{*}}$ can be written as $f=h+g$ for $h \in \ell^{1}$ and $g \in c_{0}^{\perp}$ with $\|f\|=\|h\|+\|g\|$.

Theorem 1. Let $Y \subset \ell^{\infty}$ be a subspace of codimension $n$. Then $Y$ is the range of a projection of norm one if and only if there exist $n$ different indices $t_{1}, \ldots, t_{n}$ and a basis $f_{1}, \ldots, f_{n}$ in $Y^{\perp}$ so that $f_{i}=h_{i}+g_{i}$ for $h_{i} \in \ell^{1}, g_{i} \in c_{0}^{\perp}$, for $1 \leq i \leq n$,

$$
\left\|f_{i}\right\| \leq 2\left|\left(h_{i}\right)_{t_{i}}\right| \text { and } Y=\bigcap_{1 \leq i \leq n} k e r\left(f_{i}\right) .
$$

Theorem 2. Let $Y \subset c_{0}$ be a subspace of codimension n. Then $Y$ is the range of a projection of norm one if and only if there exist $n$ different indices $t_{1}, \ldots, t_{n}$ and a basis $f_{1}, \ldots, f_{n}$ in $Y^{\perp}$ so that for $1 \leq i \leq n$,

$$
\left\|f_{i}\right\| \leq 2\left|\left(f_{i}\right)_{t_{i}}\right| \text { and } Y=\bigcap_{1 \leq i \leq n} \operatorname{ker}\left(f_{i}\right) .
$$

Theorem 3. Let $K$ be a compact Hausdorff space. Let $Y \subset C(K)$ be a subspace of codimension $n$. Then $Y$ is the range of a projection of norm one if and only if there exist $n$ distinct isolated points $t_{1}, \ldots, t_{n}$ in $K$ and a basis $\mu_{1}, \ldots, \mu_{n}$ in $Y^{\perp}$ so that for $1 \leq i \leq n$,

$$
\left\|\mu_{i}\right\| \leq 2\left|\mu_{i}\left(t_{i}\right)\right| \text { and } Y=\bigcap_{1 \leq i \leq n} k e r\left(\mu_{i}\right) .
$$

Remark 4 . Thus the spaces $c_{0}, \ell^{\infty}$ and $C(K)$ have the property (a): for any projection of norm one with finite dimensional kernel, the range is an intersection of ranges of projections of norm one with kernels of dimension one. Also of interest is property (b): finite intersections of ranges of projections of norm one with one dimensional kernel are again the range of a projection of norm one.

Thus even though one cannot expect an explicit description of such projections in general, one can still investigate spaces that have properties (a) and (or) (b). See Theorem 4.11 in 9 for more results concerning property (a) and Theorems 3.1, 3.2 and 3.3 in 8 for some results about property (b). We will be formulating our results for property (a). It is clear from the example in 4] that investigation of property (b) in general will require more information on the nature of the projections.

We now recall some concepts from $M$-structure theory from [7. We always embed $X$ in its bidaul $X^{* *}$ under the canonical embedding.

A projection $P: X \rightarrow X$ is said to be an $M(L)$-projection if

$$
\|x\|=\max \{\|P(x)\|,\|x-P(x)\|\}(\|x\|=\|P(x)\|+\|x-P(x)\|)
$$

for all $x$. The range of such a projection is called an $M(L)$-summand. These projections behave well w.r.t. projections of norm one. We recall from [7, Proposition I.1.2, that an $M$ projection is a unique norm one projection onto its range and that uniqueness of an $L$-projection is similarly determined by the kernel. 
A closed subspace $Y \subset X$ is said to be an $M$-ideal if there is an $L$-projection $P: X^{*} \rightarrow X^{*}$ such that $\operatorname{ker}(P)=Y^{\perp}$. This notion has particularly rich geometric significance when $X$ is an $M$-ideal in its bidual. For any discrete set $\Gamma, c_{0}(\Gamma)$ is an $M$-ideal in its bidual $\ell^{\infty}(\Gamma)$. See Corollary III.3.7 in [7] for several topological properties of these spaces. This property is in particular preserved by subspaces and quotients. Also, when $X$ is an $M$-ideal in its bidual, the canonical projection $Q: X^{* * *} \rightarrow X^{*}$ defined by $Q(\Lambda)=\Lambda \mid X^{*}$ is an $L$-projection and one says that $X^{*}$ is an $L$-embedded space.

We show that for a Banach space $X$ that is an $M$-ideal in its bidual, any projection of norm one in $X^{* *}$ with weak*-closed range whose kernel is finite dimensional or reflexive is the biadjoint of a similar projection in $X$. For any Banach space $X$, if in $X^{* *}$ the range of any projection of norm one with finite codimensional kernel and weak*-closed range can be written as an intersection of biadjoints of similar projections with kernels of dimension one, then a similar result is true for $X$.

We show that these properties determine $c_{0}(\Gamma)$ among preduals of $L^{1}$. We consider the property of being a central subspace of a Banach space (see [1) which is weaker than being the range of a projection of norm one. As an application of Theorem 1.1 of [2] we show that any central subspace of finite codimension in an $L^{1}$-predual space is an intersection of central hyperplanes. We also show that every central subspace in $c_{0}(\Gamma)$ and with an additional condition in $\ell^{1}$ is always the range of a projection of norm one.

\section{MAin RESUlts}

We first address the natural duality that exists between $X$ and $X^{* *}$. We recall that for any projection $P: X \rightarrow X$ with $\|P\|=1, P^{*}: X^{*} \rightarrow X^{*}$ is a projection with $\left\|P^{*}\right\|=1$, and if $Q: X^{*} \rightarrow X^{*}$ is a weak*-continuous projection (this is equivalent to the range and kernel being weak ${ }^{*}$-closed) with $\|Q\|=1$, then there exists a projection $P: X \rightarrow X$ such that $P^{*}=Q$. Further, $P^{* *}: X^{* *} \rightarrow X^{* *}$ is a projection of norm one and $P^{* *}=P$ on $X$. We call $P^{* *}$ the biadjoint of $P$. In general, ranges of projections of norm one in dual spaces need not be weak*-closed. It is known that $P$ is an $L(M)$-projection if and only if $P^{*}$ is an $M(L)$-projection.

We next prove a theorem on the structure of projections of norm one with finite dimensional kernel and weak*-closed range, for Banach spaces which are $M$-ideals in their bidual ( $M$-embedded spaces). We recall that $c_{0}$ is an $M$-ideal in its bidual $\ell^{\infty}$.

Theorem 5. Let $X$ be an $M$-ideal in its bidual. Let $P: X^{* *} \rightarrow X^{* *}$ be a projection of norm one such that the kernel of $P$ is finite dimensional and the range of $P$ is a weak $k^{*}$-closed subspace. Then there is a projection $Q: X \rightarrow X$ of norm one such that $Q^{* *}=P$. Thus weak $k^{*}$-closed subspaces of $X^{* *}$ of finite co-dimension that are ranges of projections of norm one can be completely described in terms of similar objects from $X$.

Proof. Let $x_{1}^{*}, \ldots, x_{n}^{*} \in X^{*}$ be such that $P\left(X^{* *}\right)=\bigcap_{i}^{n} k e r\left(x_{i}^{*}\right)$. Since the range and kernel of $P$ are weak*-closed subspaces, we get that $P$ is weak ${ }^{*}$-continuous. Thus there is a projection $R: X^{*} \rightarrow X^{*}$ of norm one such that $R^{*}=P$ and $\operatorname{ker}(R)=\left(\bigcap_{1}^{n} \operatorname{ker}\left(x_{1}^{*}\right)\right)^{\perp}$, where the kernels are now considered in $X$. Since $X^{*}$ is an $L$-embedded space, it follows from Proposition IV.1.5 in [7] that $R\left(X^{*}\right)$ is an $L$-embedded space. Now by Proposition IV.1.10 in [7] we get that $R\left(X^{*}\right)$ is 
weak*-closed. Therefore $R$ is weak*-continuous. Thus there exists a projection $Q: X \rightarrow X$ such that $Q^{* *}=P$.

Remark 6 . In the above proof we have used the fact that finite dimensional subspaces are always weak*-closed. Another such class of subspaces is reflexive subspaces. Recall that $Y \subset X$ is said to be factor reflexive if the quotient space $X \mid Y$ is reflexive. Arguments similar to the ones given during the proof of Theorem 5 show that if $X$ is an $M$-ideal in its bidual and $Y \subset X^{* *}$ is a weak*-closed factor reflexive subspace, then any projection $P: X^{* *} \rightarrow Y$ of norm one is the biadjoint of a projection of norm one in $X$.

Remark 7 . In the case of $\ell^{\infty}$, we recall that any complemented reflexive subspace is finite dimensional; thus we need to consider only subspaces of finite codimension.

We now formulate a general version of some of the results from 4$]$ and $[3$. Thus Theorem 1 in [3] can be seen as a bidual version of Theorem 2 (Theorem 6.3, 4]).

Theorem 8. Let $X$ be an $M$-ideal in its bidual. Suppose the range of every projection of norm one with finite dimensional kernel in $X$ can be written as an intersection of ranges of projections of norm one with kernel of dimension one. Then the range of every projection of norm one that has weak ${ }^{*}$-closed range and finite dimensional kernel in $X^{* *}$ can be written as an intersection of ranges of projections of norm one with kernel of dimension one that are biadjoints of projections in $X$. $A$ converse statement holds for any Banach space $X$.

Proof. Let $X$ be an $M$-ideal in its bidual. Let $P: X^{* *} \rightarrow X^{* *}$ be a projection of norm one with weak ${ }^{*}$-closed range and finite dimensional kernel.

From Theorem 5 we have that there is a norm one projection $Q: X \rightarrow X$ with finite dimensional kernel such that $Q^{* *}=P$. By hypothesis there exist finitely many projections $Q_{i}: X \rightarrow X$ such that $\left\|Q_{i}\right\|=1$ for each $i$ and $\operatorname{ker}\left(Q_{i}\right)$ is of dimension one and $Q(X)=\bigcap Q_{i}(X)$. Clearly $Q_{i}^{* *}$ 's have the required properties. To verify that $P\left(X^{* *}\right)=\bigcap Q_{i}^{* *}\left(X^{* *}\right)$ we note that since the operators involved are weak ${ }^{*}$-continuous and as $X$ is weak ${ }^{*}$-dense in $X^{* *}$, it is enough to check the equation at elements of $X$. Let $x \in X, P(x)=Q^{* *}(x)=Q(x)=Q_{i}\left(x_{i}\right)=Q_{i}^{* *}\left(x_{i}\right)$ for each $i$ and for some $x_{i} \in X$. Thus $P(x) \in \cap Q_{i}^{* *}\left(X^{* *}\right)$. The other direction is similarly seen.

Conversely, let $X$ be any Banach space. Let $P: X \rightarrow X$ be a projection of norm one with finite dimensional kernel. $P^{* *}: X^{* *} \rightarrow X^{* *}$ is such that $\left\|P^{* *}\right\|=1$, and it has weak*-closed range and finite dimensional kernel. Since $X^{* * *}=X^{*} \oplus X^{\perp}$ by hypothesis there exists a finite set of $x_{i}^{*} \in X^{*}$ and $\tau_{i} \in X^{\perp}$ such that $P^{* *}\left(X^{* *}\right)=$ $\bigcap \operatorname{ker}\left(x_{i}^{*}+\tau_{i}\right)$. Now since $P^{* *}\left(X^{* *}\right)$ is of finite codimension and $P^{* *}$ is weak ${ }^{*}$ continuous, we have that $P^{* *}\left(X^{* *}\right)^{\perp} \subset X^{*}$. Therefore $x_{i}^{*}+\tau_{i} \in P^{* *}\left(X^{* *}\right) \subset X^{*}$. Thus $\tau_{i}=0$ for all $i$. It is now easy to see that $P(X)=\bigcap \operatorname{ker}\left(x_{i}^{*}\right)$.

Remark 9. This explains the $c_{0} \ell^{\infty}$ duality that is evident in the results of Theorem 6.3 of 4 and the main theorem of [3]. In particular, Theorem 6.3 of 4 ] can be deduced from the main theorem of [3]. This also explains the nature of the projection in the main theorem of Baronti, [3], where the $\left(c_{0}\right)^{\perp}$ component is zero, i.e., a weak ${ }^{*}$-closed subspace of finite codimension. We conjecture that as in the main theorem of [3], for a non-reflexive $M$-embedded space $X$, finite codimensional subspaces of $X^{* *}$ that are determined only from functionals in $X^{\perp}$ cannot be the range of a projection of norm one. 
Remark 10. When $C(K)$ is a dual space or the second dual of a Banach space $X$, it seems to be difficult to interpret the conditions in Theorem 1.1 of [2] in terms of $X$, except in the case of $\ell^{\infty}(\Gamma)$. For an $L^{1}$-predual $X$, we have that $X^{* *}$ is isometric to $C(K)$ for some compact set $K$. We do not know if in $X$ the range of a projection of norm one with finite dimensional kernel is an intersection of ranges of projections of norm one with kernels of dimension one. Our approach needs projections in the second dual space to be biadjoints of projections from $X$. The results below indicate the limitations of this approach.

Our next set of results shows that the properties considered here determine $c_{0}(\Gamma)$ among $L^{1}$-preduals.

Theorem 11. Let $X$ be an $L^{1}$-predual space. Suppose every weak ${ }^{*}$-closed subspace of codimension one of $X^{* *}$ that is the range of a projection of norm one is also the range of a biadjoint projection of norm one. Then $X$ is isometric to $c_{0}(\Gamma)$.

Proof. After identifying an extreme point and its negative, in the unit ball of $X^{*}$, let $\Gamma$ denote the set of extreme points of the unit ball. For any $f \in \Gamma$, since $X$ is an $L^{1}$ predual, $\operatorname{span}\{f\}$ is an $L$-summand, i.e., $X^{*}=\operatorname{span}\{f\} \oplus_{1} N$, for some closed subspace $N$. Therefore if $Y=\operatorname{ker}(f)$, then $Y$ is an $M$-ideal and $X^{* *}=Y^{* *} \oplus_{\infty} N^{\perp}$. By hypothesis, there is a projection of norm one $P: X \rightarrow X$ with $\operatorname{ker}(P)$ one dimensional and $P^{* *}\left(X^{* *}\right)=N^{\perp}$. Since $P^{* *}$ is a projection of norm one, it now follows from our remarks in the Introduction about the uniqueness of ranges of $M$-projections that $P^{* *}$, and hence $P$, is a $M$-projection. Clearly one can choose an $x_{f} \in X$ with $f\left(x_{f}\right)=1=\left\|x_{f}\right\|$ such that $X=\operatorname{span}\left\{x_{f}\right\} \oplus_{\infty} k e r(f)$. Using the uniqueness of the range of an $M$-projection, it is easy to see that the unit vectors $\left\{x_{f}\right\}_{f \in \Gamma}$ are pair-wise independent.

Now consider the $c_{0}$-direct sum $Y=\bigoplus_{c_{0}} \operatorname{span}\left\{x_{f}\right\}$ over $\Gamma$. Clearly $Y$ is an $M$-ideal in $X$. If it is a proper $M$-ideal, $Y^{\perp}$ will be a non-trivial weak*-closed $L$-summand in $X^{*}$. Therefore there is an $f_{0} \in \Gamma$ and $Y^{\perp}$. This contradicts the fact that $f_{0}\left(x_{f_{0}}\right)=1$.

Thus $X$ is isometric to $c_{0}(\Gamma)$.

Interest in the study of ranges of projections of norm one stems from the fact that several geometric properties of the domain space are passed on to the range space. Sometimes simple geometric conditions on a subspace can achieve the same effect without the subspace actually being the range of a projection of norm one. We next recall one such notion studied in [1].

Definition 12. A closed subspace $Y \subset X$ is said to be a central subspace if for any finite set $\left\{y_{i}\right\}_{1 \leq i \leq n} \subset Y$ and $x_{0} \in X$ there exists a $y_{0} \in Y$ such that $\left\|y_{0}-y_{i}\right\| \leq\left\|x_{0}-y_{i}\right\|$ for $1 \leq i \leq n$.

It is easy to see that this is equivalent to every finite collection of balls with centres from $Y$ that intersect in $X$ also intersect in $Y$. From Lindenstrauss' characterization of $L^{1}$-preduals in terms of every collection of 4 pairwise intersecting balls having non-empty intersection (see 13, page 58), it follows that if $Y \subset X$ is a central subspace and $X$ is an $L^{1}$-predual, then $Y$ is an $L^{1}$-predual. This notion is weaker than that of an existence set considered in [12, where the inequalities in the definition should hold for all $y \in Y$. Thus the following result extends Theorem 2.3 in $[12$. 
Proposition 13. Let $Y \subset c_{0}(\Gamma)$ be a central subspace. Then $Y$ is the range of a projection of norm one.

Proof. From our remarks above we have that $Y$ is an $L^{1}$-predual.

If $X$ is finite dimensional, then $Y$ is isometric to $\ell^{\infty}(k)$ and it is well known that $Y$ is one-complemented in any superspace.

Since $Y$ is an $L^{1}$-predual, $Y^{* *}$ has the property that it is the range of a projection in any superspace (see [13], Chapter 3). Thus for the inclusion $Y^{* *} \subset \ell^{\infty}(\Gamma)$ there is a projection $P: \ell^{\infty}(\Gamma) \rightarrow Y^{* *}$ such that $\|P\|=1$. But this implies there is a projection $Q: \ell^{1}(\Gamma) \rightarrow \ell^{1}(\Gamma)$ such that $\operatorname{ker}(Q)=Y^{\perp}$ and $\|Q\|=1$. Since $c_{0}(\Gamma)$ is an $M$-embedded space, as noted during the proof of Theorem $5, Q$ is weak*continuous. Thus we get that $Y$ is the range of a projection of norm one.

The following theorem can be proved using arguments similar to the ones given earlier.

Theorem 14. Suppose $X$ is an $L^{1}$-predual space such that every central subspace of codimension one is the range of a projection of norm one. Then $X$ is isometric to $c_{0}(\Gamma)$ for some discrete set $\Gamma$.

Proof. As before, let $\Gamma$ denote the set of extreme points after identifying $f$ and $-f$. Let $f \in \Gamma$. Since $X^{*}=\operatorname{span}\{f\} \oplus_{1} N$ for a closed subspace $N$ and as $X^{*}=L^{1}(\mu)$, one has that $N$ is also an abstract $L$-space. Therefore $k e r(f)$ is an $L^{1}$-predual. It follows from Theorem 3.3 in 1 that $\operatorname{ker}(f)$ is a central subspace of $X$. Therefore by hypothesis there is a projection $P: X \rightarrow \operatorname{ker}(f)$ with $\|P\|=1$. As $k \operatorname{er}(f)$ is also an $M$-ideal, we get from Corollary I.1.3 in [7] that $P$ is an $M$-projection. Arguments similar to the ones given before now give that $X$ is isometric to $c_{0}(\Gamma)$.

We next show that certain central subspaces of $\ell^{1}$ are ranges of projections of norm one.

Proposition 15. Let $Y \subset \ell^{1}$ be a central subspace that is isometric to the range of a projection of norm one in some dual space. Then $Y$ is the range of a projection of norm one in $\ell^{1}$.

Proof. Let $X$ be a Banach space and $P: X^{*} \rightarrow X^{*}$ be a projection of norm one such that $Y$ is isometric to $\operatorname{Range}(P)$. We will show that $Y$ is an existence set. It would then follow from Theorem 2.4 in $[12$ that $Y$ is the range of a projection of norm one. Let $\alpha \in \ell^{1}$. Consider the balls $\{B(y,\|y-\alpha\|)\}_{y \in Y}$ in $Y$. Since $Y$ is a central subspace, any finite collection of balls from this family has non-empty intersection in $Y$. Ignoring the embedding of $Y$ in $X^{*}$, consider the family of closed balls in $X^{*},\left\{B_{X^{*}}(y,\|y-\alpha\|)\right\}_{y \in Y}$. Since these balls are now weak*-compact such that any finite subcollection has non-empty intersection, we have that they intersect in $Y$, and as $Y$ is the range of a projection of norm one, there is a $y_{0}$ such that $\left\|y-y_{0}\right\| \leq\|y-\alpha\|$ for all $y \in Y$. Thus $Y$ is an existence set.

Remark 16. In particular, we have that any weak ${ }^{*}$-closed (w.r.t. any predual of $\ell^{1}$ ) central subspace of $\ell^{1}$ is the range of a projection of norm one. We do not know if a central subspace of $\ell^{1}$ is always the range of a projection of norm one.

Since the range of a projection of norm one is a central subspace, the following result is an application of Theorem 1.1 in [2]. 
Theorem 17. Let $X$ be an $L^{1}$-predual space. Any central subspace of finite codimension is the intersection of hyperplanes that are central subspaces.

Proof. Let $Y \subset X$ be a central subspace and $Y=\bigcap_{1<i<n} k e r\left(f_{i}\right)$ for $f_{i} \in X^{*}$. We denote by $\hat{f}_{i}$ the canonical image of $f_{i}$ in $X^{* * *}$. As before, $Y^{\perp \perp}=\bigcap_{1 \leq i \leq n} \operatorname{ker}\left(\hat{f}_{i}\right)$. We have that $Y^{\perp \perp} \subset X^{* *}$ and $X^{* *}$ is isometric to $C(K)$ for some compact extremally disconnected space $K$ (see [11, Section 11). As noted before, $Y$ is also an $L^{1}$-predual space, and thus $Y^{* *}=Y^{\perp \perp}$ is isometric to $C\left(K^{\prime}\right)$ for some compact extremally disconnected space $K^{\prime}$. It follows from Corollary 2 in Section 11 of 11 that $Y^{\perp \perp}$ is the range of a projection of norm one in $X^{* *}$. It now follows from Theorem 1.1 of [2] that for $1 \leq i \leq n$ there exists a projection of norm one, $P_{i}: C(K) \rightarrow \operatorname{ker}\left(\hat{f}_{i}\right)$. This in particular implies that $\operatorname{ker}\left(f_{i}\right)$ is an $L^{1}$-predual space.

We now show that $\operatorname{ker}\left(f_{i}\right)$ is a central subspace of $X$. Let $y_{1}, \ldots, y_{k} \in \operatorname{ker}\left(f_{i}\right)$, $x \in X$ and $\epsilon>0$. By the principle of local reflexivity applied to the subspace $\operatorname{span}\left\{y_{1}, \ldots, y_{k}, P_{i}(x)\right\} \subset \operatorname{ker}\left(\hat{f}_{i}\right)$, there is an operator $T: \operatorname{ker}\left(\hat{f}_{i}\right) \rightarrow \operatorname{ker}\left(f_{i}\right),\|T\| \leq$ $1+\epsilon$ such that $T\left(y_{j}\right)=y_{j}$ for $1 \leq j \leq k$. Thus for $1 \leq j \leq k$,

$$
\begin{aligned}
\left\|T\left(P_{i}(x)\right)-y_{j}\right\| & =\left\|T\left(P_{i}(x)-y_{j}\right)\right\| \leq(1+\epsilon)\left\|P_{i}(x)-y_{j}\right\| \\
& =\left\|P_{i}\left(x-y_{j}\right)\right\| \leq(1+\epsilon)\left\|x-y_{j}\right\| .
\end{aligned}
$$

As $k e r\left(f_{i}\right)$ is an $L^{1}$-predual space, by Lemma 4.2 in 13 it follows that there is a $y \in \operatorname{ker}\left(f_{i}\right)$ such that $\left\|y-y_{j}\right\| \leq\left\|x-y_{j}\right\|$ for $1 \leq j \leq k$. Therefore $\operatorname{ker}\left(f_{i}\right)$ is a central subspace.

Corollary 18. Let $X$ be an $L^{1}$-predual space. Let $Y \subset X$ be a central subspace of codimension $n$. For any $1 \leq k<n$ there is a central subspace $Z$ of codimension $k$ with $Y \subset Z \subset X$.

Proof. It follows from the above theorem that $Y^{\perp \perp}=\bigcap_{1 \leq i \leq n} \operatorname{ker}\left(\hat{f}_{i}\right)$, where the $\hat{f}_{i}$ 's satisfy condition ii) in part (c) of Theorem 1.1 in [2]. Now by Theorem 1.1 of [2] again, for any $1 \leq k<n, \bigcap_{1 \leq i \leq k} \operatorname{ker}\left(\hat{f}_{i}\right)$ is the range of a projection of norm one in $X^{* *}$. Thus $Z=\bigcap_{1 \leq i \leq k} \operatorname{ker}\left(\bar{f}_{i}\right)$ is a central subspace and $Y \subset Z \subset X$.

\section{ACKNOWLEDGEMENTS}

Part of this work was done during the author's stay at the University of Bologna during June 2011 under the continuation of an India4EU Fellowship. He thanks the Department of Mathematics and Professor P. L. Papini for their hospitality. He also thanks Professor K. Jarosz and the Department of Mathematics at S.I.U.E., where he was a Fulbright-Nehru Senior Research Fellow from September 2011 to May 2012, for their hospitality.

\section{REFERENCES}

[1] P. Bandyopadhyay and T. S. S. R. K. Rao, Central subspaces of Banach spaces, J. Approx. Theory 103 (2000), 206-222. MR1749962(2001b:46022)

[2] P. Bandyopadhyay and S. Dutta, Almost constrained subspaces of Banach spaces. II, Houston J. Math. 35 (2009), 945-957. MR2534290 (2011a:46023)

[3] M. Baronti, A note on norm-one projections onto subspaces of finite codimension of $l^{\infty}$, Arch. Math. (Basel) 54 (1990), 384-388. MR1042133 (91c:46029)

[4] M. Baronti and P. Papini, Norm-one projections onto subspaces of finite codimension in $l_{1}$ and $c_{0}$, Period. Math. Hungar. 22 (1991), 161-174. MR.1142506 (93a:46034) 
[5] J. Blatter and E. W. Cheney, Minimal projections on hyperplanes in sequence spaces, Ann. Mat. Pura Appl. 101 (1974), 215-227. MR0358179 (50:10644)

[6] A. P. Bosznay and B. M. Garay, On norms of projections, Acta Sci. Math. (Szeged) 50 (1986), 87-92. MR.862183(88d:47012)

[7] P. Harmand, D. Werner and W. Werner, M-ideals in Banach spaces and Banach algebras, Lecture Notes in Mathematics, 1547. Springer-Verlag, Berlin, 1993. MR1238713 (94k:46022)

[8] J. E. Jamison, A. Kamińska and G. Lewicki, One-complemented subspaces of Musielak-Orlicz sequence spaces, J. Approximation Theory 130 (2004), 1-37. MR2086807 (2005f:46035)

[9] A. Kamińska and G. Lewicki, Contractive and optimal sets in modular spaces, Math. Nachr. 268 (2004), 74-95. MR2054533 (2005c:46033)

[10] A. Kamińska, H. J. Lee and G. Lewicki, Extreme and smooth points in Lorentz and Marcinkiewicz spaces with applications to contractive projections, Rocky Mountain J. Math. 39 (2009), 1533-1572. MR2546654 (2010k:46014)

[11] H. E. Lacey, The isometric theory of classical Banach spaces, Die Grundlehren der mathematischen Wissenschaften, Band 208. Springer-Verlag, New York-Heidelberg, 1974. MR0493279 $(58: 12308)$

[12] G. Lewicki and G. Trombetta, Optimal and one-complemented subspaces, Monatsh. Math. 153 (2008), 115-132. MR2373365 (2010a:46073)

[13] J. Lindenstrauss, Extension of compact operators, Mem. Amer. Math. Soc. No. 48 (1964), 112 pp. MR0179580 (31:3828)

Theoretical Statistics and Mathematics Unit, Indian Statistical Institute, R. V. College P. O., Bangalore 560059, India

E-mail address: tss@isibang.ac.in 